\title{
Amadeus
}

International Multidisciplinary Journal ISSN 2525-8281

V.2, N. 4. Jul./2018 - ISSN 2525-8281

\section{EFFECTIVENESS AND INTERVENTIONS OF THE MUSIC THERAPY IN PATIENTS IN PALLIATIVE CARE}

Kevellyn Cruz Aguilera ${ }^{\text {, }}$ Antonio Marlos Duarte de

Melo $^{2}$,

Brenda Lacerda da Silva', Leyde Jenifer Dias Uchôa, Rivânia Beatriz Novais Lima ${ }^{1}$, Bruna Figueiredo Medeiros ${ }^{1}$, Esther de Macêdo Lira', Amon Vitorino Duarte ${ }^{1}$, Ricardo Souto Quidute ${ }^{1}$

\begin{abstract}
Currently, music therapy, as a low-cost therapeutic approach, has been successfully applied in palliative care patients, aiming to improve emotional stress and mood. Besides these advantages, the music therapy can influence the patient to have a better social interaction. In cancer patients, especially, music therapy associated with massage therapy is very costeffective, pointing to the need for the formulation of other nonpharmacological methods. Music is intrinsically related to the reduction of pain, anxiety and stress, as well as a direct relationship with spiritual improvement and quality of life. Music therapy reinforces multidisciplinary care for the patient in palliative care, and may even reduce pain in those affected.
\end{abstract}

Keywords: Music therapy; Palliative care; Multidisciplinary Care.

\section{Introduction}

Palliative care, which spread in the 1970s in Canada, involves a multidisciplinary team that is activated when there are cardiovascular diseases, cancer, chronic respiratory diseases, renal failure, rheumatoid arthritis, dementias, tuberculosis with resistance to therapeutic schemes and diabetes, focusing on pain relief, fatigue and stress reduction, with consequences on the patient's quality of life.

\footnotetext{
${ }^{1}$ Faculty of Medicine Estacio of Juazeiro do Norte, Juazeiro do Norte, Ceara, Brazil.

${ }^{2}$ City Hall of Custodia, Custodia, Pernambuco, Brazil.

Corresponce Author: Kevellyn Cruz Aguilera. Estacio-FMJ. Medicine course.

Tenente Raimundo Rocha Street, 515, Cidade Universitaria, Juazeiro do Norte, Ceara, Brazil kevellynaguilera@hotmail.com 
There are complementary techniques to the conventional therapy, but most of them with low cost-benefit, pointing out the necessity of the formulation of other methods with lower cost and better effectiveness, such as music therapy, which together with massotherapy make up, in greater proportion, the forms of non-pharmacological treatment, especially in cancer patients, in the palliative care units. ${ }^{1,2,3,4}$

Music is seen as an instrument for reducing pain, anxiety and stress and improving quality of life, both in the physical, emotional and spiritual domain, being used not only in palliative care but also in other areas, such as geriatrics and psychiatry. Therefore, it is an alternative to compose the therapeutic arsenal that can be performed, according to the patient's profile and goal setting, in two main ways: one is the active one, in which the patient hears the sound and the other would be receptive, in which the patient can either create or reproduce the songs of his or her preference. ${ }^{4,5,6}$

In music therapy there is the aspect of emotion induction and its positive modulation, directly impacting the mood, which generates the distraction of physical and emotional suffering, linking therapy to happy reminiscences and helping to recover, at this juncture, their joy and reducing the negativity against their condition. It has been demonstrated, in general, a decrease of the tension levels and an improvement of the patient's well-being, however it is necessary to evaluate that, according to the music therapist and with the different methods of music therapy, there is promotion of distinct responses and positive perception of music therapy. Thus, music therapy can be used in its therapeutic arsenal, and the patient can be questioned and choose whether or not to use this tool. ${ }^{4,6,7}$

\section{Music therapy}

Music therapy is used, with a gradual increase, for more than 35 years in palliative care, both at the hospital and outpatient levels, as a systematic process that contains songs and sounds in a dynamic way, involving the music therapist and the patient, and, as a consequence improves the spiritual aspects, especially in a welcoming way, between the doctor and the patient. In addition, improvement of clinical parameters such as heart and 
respiratory rates, decrease in pressure levels and improvement of mood aspects were observed safely and without side effects. . $^{8,9} 10$

The association between palliative care and music therapy addresses the patient in its entirety with a multidisciplinary approach, which, when evaluating the patient, understands their specific objectives, contributing to provide more comfort to both patients and their families. Thus, patients feel more valued, especially when they perform some function within the therapy such as playing an instrument, giving greater purpose to their own life and providing opportunities to find peace. ${ }^{4,6,11}$

Patients with incurable diseases and with low life expectancy do not fit the standards of curative medicine, becoming vulnerable and requiring the palliative care that directs therapies to increase the quality of life of both patients and their families, according to the profile of each patient. This leads to improvements in the quality of life, encompassing emotional, physical and spiritual aspects. ${ }^{3,12,13}$

The specific demands of each patient are stratified according to the patient and his / her family, as it is analyzed, before starting the session by the music therapist, how this therapy can be performed to achieve a better impact on quality of life to deal with both physical pain emotional consequences of the bad news shared for the patient. It is a medicine that is person-centered, holistic, and not just in disease as it was in the case of the biomedical technique, which fragments the individual according to the pathology, aiming only for healing and not for comfort. ${ }^{6,7,13}$

Patients in palliative care often have too much pain that does not stop well with medication, but adjunctive therapies such as music therapy can more effectively alleviate some symptoms, such as fatigue, depression, and relatively common problems in this population. to evoke memories of pleasure and joy associated with the songs that make up the therapeutic arsenal. ${ }^{1,7,8}$

It is a challenge for medical staff to deal with the pain, concerns, and reactions that come with the diagnosis. Considering this context, palliative care, associated with music therapy, acts in situations where the therapeutic part for the underlying disease is exhausted, helping to reduce pain and stress, and to recover the sense of familiarity. Moreover, this therapy can regulate negative emotions and improve communication, which impacts on 
greater social engagement, reducing social isolation rates, which can lead to depression in the patient. ${ }^{1,5,6}$

Music therapy, unlike a medicine that acts specifically in an illness, deals with the integrality of the individual receiving the treatment, responding to the different realities encountered by each one. However, in the scientific field, music therapy has few articles with statistically significant numbers and many researches are performed without randomization and without control groups, with small population samples, which leads to the indispensability of building more articles that approach the theme. ${ }^{12,13}$

\section{Conclusion}


Therefore, in this perspective of holistic care, the use of music therapy for the reduction of anxiety and stress levels is shown as an auxiliary therapy, which, in association with other treatments, is cost-effective and requires further studies to confirm the advantages of this modality in palliative care, aiming to increase and improve the quality of life. Further studies on the importance of music therapy in the adjuvant treatment of the patient in palliative care are expected in order to avoid the use of unnecessary medications that would be prescribed to combat stress and anxiety. This approach also helps to recover the familiarity, the state of humor and to improve the communication of the patient, obtaining, as a result, a lower social isolation,

\section{References}

1. Archie P.; Bruera E.; Cohen L. Music-based interventions in palliative cancer care: a review of quantitative studies and neurobiological literature. Support Care Cancer. 2013, 21(9):2609-24. https://doi.org/10.1007/s00520-013-1841-4.

2. Coelho, Adriana et al. Use of non-pharmacological interventions for comforting patients in palliative care. Jbi Database of Systematic Reviews and Implementation Reports, 2017, v. 15, n. 7, p.1867-1904, jul. Ovid Technologies (Wolters Kluwer Health). http://dx.doi.org/10.11124/jbisrir-2016-003204.

3. World Health Organization. Palliative Care. http://www.who.int/en/news-room/factsheets/detail/palliative-care, 2018.

4. Warth, Marco et al. Music therapy in palliative care - a randomized controlled trial to evaluate effects on relaxation. Dtsch Arztebl Int, 2015, 112: 788-94. https://www.ncbi.nlm.nih.gov/pmc/articles/PMC4671329/.

5. Krishnaswamy P.; Nair S. Effect of music therapy on pain and anxiety levels of cancer patients: A pilot study. Indian $J$ Palliat Care, 2016, 22:307-11. https://www.ncbi.nlm.nih.gov/pubmed/27559260.

6. Porter, Sam et al. A critical realist evaluation of a music therapy intervention in palliative care. Bmc Palliative Care, 2017, v. 16, n. 1, p.1-2, dez. Springer Nature. http://dx.doi.org/10.1186/s12904-017-0253-5.

7. Ramirez, Rafael et al. EEG-Based Analysis of the Emotional Effect of Music Therapy on Palliative Care Cancer Patients. Frontiers in Psychology. 2018, 9:254. https://www.ncbi.nlm.nih.gov/pmc/articles/PMC5840261/. 
8. Preissler, Pia et al. Favored subjects and psychosocial needs in music therapy in terminally ill cancer patients: a content analysis. BMC palliative care, 2016, 15, 48. https://bmcpalliatcare.biomedcentral.com/articles/10.1186/s12904-016-0122-7.

9. Teut, Michael et al. Perceived outcomes of music therapy with body tambura in end of life care - a qualitative pilot study. BMC Palliat Care. 2014, 13(1):18. https://www.ncbi.nlm.nih.gov/pmc/articles/PMC3986446/.

10. Warth, Marco et al. Music therapy to promote psychological and physiological relaxation in palliative care patients: protocol of a randomized controlled trial. Bmc Palliative Care, 2014, v. 13, n. 1, p.1-7, dez. Springer Nature. http://dx.doi.org/10.1186/1472-684x-13-60.

11. Mcconnell, Tracey et al. Evaluation of the effectiveness of music therapy in improving the quality of life of palliative care patients: a randomised controlled pilot and feasibility study. Pilot And Feasibility Studies, 2016, v. 2, n. 1, p.1-8, 29 nov. Springer Nature. http://dx.doi.org/10.1186/s40814-016-0111-x.

12. Korczak D.; Wastian M.; Schneider M. Music therapy in palliative setting. GMS Health Technol Assess. 2013, 9:Doc07. http://doi.org/10.3205/hta000113

13. Schmid, W. et al. Patient's and health care provider's perspectives on music therapy in palliative care - an integrative review. BMC Palliative Care. https://www.ncbi.nlm.nih.gov/pmc/articles/PMC5819707/, 2018.

\section{How to cite this article (APA format):}

Aguilera, Kevellyn Cruz; Melo, Antonio Marlos Duarte de; Silva, Brenda Lacerda da; Uchôa, Leyde Jenifer Dias; Lima, Rivânia Beatriz Novais; Medeiros, Bruna Figueiredo; Lira, Esther de Macêdo; Duarte, Amon Vitorino; Quidute, Ricardo Souto. (2018). Effectiveness and Interventions of the Music Therapy in Patients in Palliative Care. Am In. Mul J, 2 (4), p. 100-105.

Received: 07/04/2018.

Accepted: 07/06/2018. 